\title{
The Degree of Disease Knowledge in Patients with Gastroesophageal Reflux Disease: A Multi-center Prospective Study in Korea
}

\begin{abstract}
In Du Jeong, ${ }^{1}$ Moo In Park ${ }^{2 *}$ Sung Eun Kim, ${ }^{2}$ Beom Jin Kim, ${ }^{3}$ Sang Wook Kim, ${ }^{4}$ Jie-Hyun Kim, ${ }^{5}$ Hye Young Sung, ${ }^{6}$ Tae-Hoon Oh, and Yeon Soo $\mathrm{Kim}^{8}$; The Korean Society of Neurogastroenterology and Motility, GERD and Esophageal Function Research Committee

${ }^{1}$ Department of Internal Medicine, Ulsan University Hospital, University of Ulsan College of Medicine, Ulsan, Korea; ${ }^{2}$ Department of Internal Medicine, Kosin University College of Medicine, Busan, Korea; ${ }^{3 D}$ epartment of Internal Medicine, Chung-Ang University College of Medicine, Seoul, Korea; ${ }^{4}$ Department of Internal Medicine, Chonbuk National University Medical School, Jeonju, Jeollabuk-do, Korea; ${ }^{5}$ Department of Internal Medicine, Yonsei University College of Medicine, Seoul, Korea; ${ }^{6}$ Department of Internal Medicine, Wonkwang University College of Medicine, Iksan, Jeollabuk-do, Korea; ${ }^{7}$ Department of Internal Medicine, Inje University College of Medicine, Seoul, Korea; and ${ }^{8}$ Department of Internal Medicine, Hallym University College of Medicine, Chuncheon, Gangwon-do, Korea
\end{abstract}

\section{Background/Aims}

Patient education has been shown to be beneficial in several diseases. To properly educate patients with gastroesophageal reflux disease (GERD), it is necessary to understand how much they already know about their disease. However, no study has examined the degree of disease knowledge in Korean patients with GERD. Therefore, we conducted this study to assess the degree of knowledge in such patients.

\section{Methods}

This multicenter prospective study was conducted from January 2014 to January 2015. A total of 746 patients (mean age, 52 years; $57.6 \%$ female) were enrolled from 7 hospitals in Korea. Inclusion criteria were diagnosis of GERD and ability to properly complete a survey. Degree of disease knowledge was assessed using the translated, validated Korean Urnes questionnaire, which consists of 22 items related to GERD.

\section{Results}

Mean percentage of correct answers was 46.3\% and mean GERD knowledge score was 9.6. Degree of knowledge (mean percentage of correct answers) regarding etiology, prognosis, and treatment of GERD were $49.5 \%, 36.7 \%$, and $37.5 \%$, respectively. Degree of disease knowledge differed significantly according to age $(P<0.001)$, education $(P<0.001)$, income $(P=0.028)$, and occupation $(P<0.001)$. In multivariate analysis, using multiple logistic regression, the higher knowledge score group tended to have higher education and professional occupation.

\section{Conclusions}

The surveyed Korean patients had relatively low disease knowledge, suggesting that a GERD educational program may be beneficial in Korea. Formulation of a program is underway.

(J Neurogastroenterol Motil 2017;23:385-391)

Key Words

Gastroesophageal reflux; Knowledge; Korea; Surveys and questionnaires

Received: August 8, 2016 Revised: February 13, 2017 Accepted: February 16, 2017

(.) This is an Open Access article distributed under the terms of the Creative Commons Attribution Non-Commercial License (http://creativecommons. org/licenses/by-nc/4.0) which permits unrestricted non-commercial use, distribution, and reproduction in any medium, provided the original work is properly cited.

*Correspondence: Moo In Park, MD

Department of Internal Medicine, Kosin University College of Medicine, 262 Gamcheon-ro, Seo-gu, Busan 49267, Korea Tel: +82-51-990-6719, Fax: +82-51-990-5055, E-mail: mipark@ns.kosinmed.or.kr 


\section{Introduction}

Prevalence of gastroesophageal reflux disease (GERD) and incidence of its complications have increased remarkably over the last few decades. There is evidence of ethnic variation in $\mathrm{GERD}^{1}$ : overall prevalence of GERD is reported to be $10 \%$ to $20 \%$ in Western countries, ${ }^{2-4}$ but only $2.5 \%$ to $8.5 \%$ in Asian countries. ${ }^{5-7}$ However, recent studies have shown increasing prevalence of GERD in Asia countries. ${ }^{8}$ In South Korea, the recent prevalence of GERD was $7.1 \%$. ${ }^{9}$ Patient education has been shown to be beneficial in some chronic diseases. ${ }^{10-14}$ Evaluation of a patient education program related to GERD found improvement in quality of life in patients who had completed primary school, but not in patients who had advanced education. ${ }^{15}$ In order to properly educate patients with GERD, it is necessary to understand how much they already know about their disease. However, no study has examined the degree of disease knowledge in Korean patients with GERD. Therefore, this study was conducted to assess the degree of disease knowledge in such patients, with the aim of developing a meaningful GERD educational program.

\section{Materials and Methods}

From January 2014 to January 2015, we conducted a prospective survey of 746 Korean patients with GERD aged 15 to 91 years in order to assess their degree of disease knowledge. This study was conducted by the GERD study group of the Korean Society of Neurogastroenterology and Motility, which includes members from multiple centers in South Korea. The study was approved by the institutional review boards of the 7 hospitals that were involved. All of the patients enrolled voluntarily and provided written informed consent for participation. For study inclusion, patients had to be diagnosed as having GERD, which was defined as follows: (1) typical GERD symptoms dominated by heartburn and/or acid regurgitation; (2) positive response to proton pump inhibitor administration; and/or (3) diagnosis of erosive reflux disease based on upper endoscopic examination. Exclusion criteria were lack of consent and inability for any reason to complete the survey. The structured survey was composed of 2 sections: demographics and GERD knowledge. The GERD knowledge section consisted of 22 statements regarding GERD. Degree of disease knowledge was assessed using the translated Korean version (Supplementary Table) of the Urnes' descriptive survey items (Appendix). ${ }^{16}$ In the development of the Korean version of the questionnaire, we first asked for the original author's permission via email. Upon permission, the original instrument was translated, back-translated, and tested for reproducibility using a sample of patients and nurses. The original author validated the translated version. Respondents answered each statement as "true," "false," or “don't know." GERD knowledge score was the sum of correct responses (range 0-22). We also surveyed the overall knowledge of patients with GERD by using a questionnaire consisting of closed-ended questions.

\section{Statistical Methods}

Clinical and sociodemographic variables were analyzed using SPSS 17.0 (IBM Corp, Armonk, NY, USA). Associations between disease knowledge and sociodemographic variables at baseline were analyzed based on bivariate correlations. Associations involving parametric data were assessed using the Student's $t$ test, while dichotomous nonparametric data were assessed using the Chi-square test or Fisher's exact test. Multivariate analysis was performed using the variables associated with high disease knowledge in univariate analysis $(P<0.05)$. Odds ratios $(\mathrm{ORs})$ were presented together with $95 \%$ confidence intervals (CIs). $P$-values of $<0.05$ were considered statistically significant.

\section{Results}

\section{Patient Characteristics}

Table 1 summarizes the baseline characteristics of the patients. A total of 746 patients completed the survey, which was administered onsite at 7 hospitals in Korea. Mean age of the patients was

Table 1. Baseline Characteristics of Patients with Gastroesophageal Reflux Disease

$\begin{array}{lc}\text { Age (median age [range], yr) } & 52(15-91) \\ \text { Gender (n [\%]) } & 316(42.4) \\ \quad \text { Male } & 430(57.6) \\ \quad \text { Female } & \\ \text { Education (n [\%]) } & 447(59.9) \\ \quad \leq \text { High school } & 299(40.1) \\ \quad \geq \text { College } & \\ \text { Monthly income (n [\%], \$) } & 443(59.4) \\ <\$ 3000 & 261(35.0) \\ \quad \$ 3000-\$ 6000 & 42(5.6) \\ >\$ 6000 & \\ \text { Occupation (n [\%]) } & 392(52.6) \\ \text { Employed } & 233(31.2) \\ \text { Housewife } & 121(16.2) \\ \text { Unemployed or retired } & \end{array}$


52 years (range, 15-91 years), and 57.6\% were female. Regarding education, 447 patients (59.9\%) had not completed college, while 299 (40.1\%) had completed college. Regarding income, 443 (59.4\%) earned less than $\$ 3000$ per month, while 42 (5.6\%) earned more than $\$ 6000$ per month. A total of 392 patients (52.6\%) had a professional occupation, $233(31.2 \%)$ were housewives, and 121 (16.2\%) were retired or unemployed.

\section{Closed-ended Questionnaire Results}

Many patients reported an interest in learning about medical or nonmedical treatment and etiology of GERD (Table 2). Many patients also reported that they knew about GERD from a physician, internet, television, or brochure, with these sources of information regarded as being the most important to obtain knowledge of GERD.

\section{Gastroesophageal Reflux Disease Knowledge Test Results}

Mean percentage of correct answers was $46.3 \%$ (Table 3).

\section{Symptoms and etiology}

Twenty-two percent of patients correctly believed that bloating
Table 2. Answer for Closed-ended Questionnaire on Gastroesophageal Reflux Disease

\begin{tabular}{lc}
\hline & $\begin{array}{c}\text { Number of } \\
\text { multiple responses }\end{array}$ \\
\hline What do you want to know about GERD? & 252 \\
Etiology & 65 \\
Diagnostic method & 179 \\
Medical treatment & 243 \\
Non-medical treatment & 116 \\
Prognosis & \\
From where did you first hear about GERD? & 130 \\
Television & 20 \\
Newspaper & 5 \\
Magazine & 156 \\
Internet & 47 \\
Other patients & 384 \\
Physician & 101 \\
Brochure & \\
Which method do you think is the best to & \\
obtain GERD information? & 83 \\
Television/internet/radio & 15 \\
Newspaper & 376 \\
Magazine & 252 \\
Physician & \\
Brochure & \\
\hline
\end{tabular}

GERD, gastroesophageal reflux disease.

Table 3. Knowledge Degree of Gastroesophageal Reflux Disease in Subjects

\begin{tabular}{|c|c|}
\hline Questions & Correct answer, $\mathrm{n}(\%)$ \\
\hline Bloating is a symptom in reflux disease & False, 165 (22) \\
\hline Cough may be a symptom of reflux disease & True, $304(40)$ \\
\hline Difficulties with swallowing may occur in reflux disease & True, $379(50)$ \\
\hline \multicolumn{2}{|l|}{ Which of the following occurs during an episode of reflux? } \\
\hline The sphincter muscle between the stomach and the esophagus relaxes & True, 348 (46) \\
\hline The esophagus tightens & False, $193(25)$ \\
\hline Acid leaks from the stomach into the esophagus & True, 489 (65) \\
\hline The production of bile increases & False, 142 (19) \\
\hline Small meals will often increase reflux & False, $321(43)$ \\
\hline Fatty foods will often increase reflux & True, $452(60)$ \\
\hline Coffee will often aggravate reflux & True, $559(75)$ \\
\hline Late night meals may stimulate reflux & True, $593(79)$ \\
\hline Bending forwards may worsen reflux & True, 321 (43) \\
\hline Nervousness is a cause of reflux disease & False, $70(9)$ \\
\hline Sedatives are an important treatment in reflux disease & False, 215 (28) \\
\hline Medicine stimulating intestinal motility is used against reflux disease & True, $199(26)$ \\
\hline Acid production inhibitors are used against reflux disease & True, 417 (56) \\
\hline Reflux may inflict ulcer in the esophagus & True, 497 (66) \\
\hline Reflux may inflict ulcer in the stomach & False, 124 (16) \\
\hline Constriction of the esophagus may occur as a consequence of reflux & True, $310(41)$ \\
\hline Reflux disease may lead to heart disease & False, 205 (27) \\
\hline Blood tests may be used to prove a diagnosis of reflux & False, 237 (32) \\
\hline Gastroscopy is an important investigation in reflux disease & True, $650(87)$ \\
\hline
\end{tabular}


is not a symptom of reflux disease, $50 \%$ correctly believed that difficulty swallowing may occur in reflux disease, $63.1 \%$ falsely believed that nervousness is a cause of reflux disease, and only $9 \%$ answered all of these items correctly. Regarding GERD exacerbating factors, $60 \%, 75 \%$, and $79 \%$, respectively, correctly believed that fatty foods, coffee, and late night meals may exacerbate reflux.

\section{Management}

Twenty-eight percent of patients correctly believed that sedatives are not an important treatment for reflux disease, while $56 \%$ correctly believed that acid production inhibitors are used for management of reflux disease.

\section{Prognosis}

Sixty-six percent of patients correctly believed that serious heartburn can lead to esophageal ulcers, whereas $84 \%$ falsely believed that reflux may cause ulcers in the stomach. Twelve percent of patients falsely believed that serious heartburn can lead to heart disease, whereas only $27 \%$ knew that this association does not exist.

Table 4. Sociodemographic Characteristics Between Higher Score Group and Lower Score Group

\begin{tabular}{|c|c|c|c|}
\hline Variables & $\begin{array}{c}\text { Score } \geq 9.6 \\
(n=386)\end{array}$ & $\begin{array}{c}\text { Score }<9.6 \\
(n=360)\end{array}$ & $P$-value \\
\hline Mean age $(y r)^{a}$ & 50.4 & 54.1 & $<0.001$ \\
\hline $\operatorname{Sex}(M: F)^{b}$ & $157: 229$ & $159: 201$ & 0.335 \\
\hline Education $^{\mathrm{b}}$ & & & $<0.001$ \\
\hline Elementary & 35 & 48 & \\
\hline Middle school & 52 & 70 & \\
\hline High school & 111 & 131 & \\
\hline College & 188 & 111 & \\
\hline Monthly income ${ }^{b}$ & & & 0.028 \\
\hline$<\$ 3000$ & 216 & 227 & \\
\hline$\$ 3000-\$ 6000$ & 141 & 120 & \\
\hline$>\$ 6000$ & 29 & 13 & \\
\hline Occupation $^{\mathrm{b}}$ & & & $<0.001$ \\
\hline Housewife & 115 & 118 & \\
\hline Seller & 14 & 23 & \\
\hline Administrator & 35 & 32 & \\
\hline Profession & 106 & 43 & \\
\hline Service worker & 38 & 31 & \\
\hline Agriculture & 9 & 20 & \\
\hline Engineer & 5 & 10 & \\
\hline Simple worker & 8 & 9 & \\
\hline Soldier & 3 & 6 & \\
\hline Unemployed or retired & 53 & 68 & \\
\hline
\end{tabular}

${ }^{a}$ Independent $t$ test (or Student's $t$ test).

${ }^{\text {b} P e a r s o n ' s ~ C h i-s q u a r e ~ t e s t . ~}$

\section{Factors associated with disease knowledge}

Most patients (87\%) were correct in identifying gastroscopy as an important investigation in reflux disease. The statement with the lowest correct answer rate (9\%) was that nervousness is a cause of reflux disease. Mean GERD knowledge score was 9.6. We then assessed the differences with regard to sociodemographic variables between the higher score group (mean score $\geq 9.6, \mathrm{n}=386$ ) and the lower score group (mean score $<9.6, \mathrm{n}=360$ ). Table 4 shows the sociodemographic characteristics between these 2 groups. Degree of disease knowledge differed significantly according to age $(P<0.001)$, education $(P<0.001)$, income $(P=0.028)$, and occupation $(P<0.001)$. In multivariate analysis, using multiple logistic regression, the higher knowledge score group tended to have college education (OR, 1.763; 95\% CI, 1.009-3.082; $P=0.047$ ) and professional occupation (OR, 2.619; 95\% CI, 1.550-4.426; $P$ $<0.001)$ (Table 5).

\section{Discussion}

We conducted this study to investigate the degree of disease knowledge in Korean patients with GERD in order to provide baseline data for formulation of a proper and meaningful GERD educational program in Korea. Increased disease knowledge will not only alert patients regarding symptoms, that may be indicative of GERD, but also make them aware of necessary behavioral changes, including diet, substance use, knowing how to avoid and reduce the severity of symptoms, taking appropriate medications, and seeking professional care at an earlier time.

In this study, the mean percentage of correct answers was $46.3 \%$, and the mean GERD knowledge score was 9.6 (range, 0 -22). These findings are lower than those of Urnes et al, ${ }^{16}$ who reported a mean GERD knowledge score of 13.1 at 2 months and 14.0 at 12 months in patients allocated to a control group (range, 0 -24). These findings may suggest that degree of GERD knowledge in Korea is lower than that of Western countries. However, the present study was relatively large, with 746 patients completing the onsite survey, whereas Urnes et $\mathrm{al}^{16}$ enrolled only 200 subjects. In addition, concerning the translated questionnaire, differences in culture and language may have influenced the lower GERD knowledge score in Korean patients. Further research is required to explore GERD knowledge in other countries.

The majority of patients correctly answered that fatty foods, late night meals, and coffee may increase reflux. However, $78 \%$ falsely believed that bloating is a symptom of reflux disease and $91 \%$ falsely believed that nervousness is a cause of reflux disease. These find- 
Table 5. Result of Multivariable Logistic Regression Analysis of Factors Associated with Disease Knowledge

\begin{tabular}{|c|c|c|c|c|c|c|}
\hline \multirow{2}{*}{ Variables } & \multirow{2}{*}{ B } & \multirow{2}{*}{$\mathrm{SE}$} & \multirow{2}{*}{ Odds } & \multicolumn{2}{|c|}{$95 \%$ CI Odds } & \multirow{2}{*}{$P$-value } \\
\hline & & & & Lower & Upper & \\
\hline Constant & -0.489 & 0.288 & 0.613 & & & 0.089 \\
\hline \multicolumn{7}{|l|}{ Education } \\
\hline Elementary & \multicolumn{6}{|c|}{ Reference } \\
\hline Middle school & -0.065 & 0.294 & 0.937 & 0.526 & 1.668 & 0.825 \\
\hline High school & 0.004 & 0.271 & 1.004 & 0.590 & 1.706 & 0.990 \\
\hline College & 0.567 & 0.285 & 1.763 & 1.009 & 3.082 & 0.047 \\
\hline \multicolumn{7}{|l|}{ Occupation } \\
\hline Unemployed or retired & \multicolumn{6}{|c|}{ Reference } \\
\hline Housewife & 0.390 & 0.234 & 1.477 & 0.934 & 2.335 & 0.095 \\
\hline Seller & -0.158 & 0.391 & 0.854 & 0.397 & 1.835 & 0.685 \\
\hline Administrator & 0.208 & 0.314 & 1.231 & 0.665 & 2.278 & 0.508 \\
\hline Profession & 0.963 & 0.268 & 2.619 & 1.550 & 4.426 & 0.000 \\
\hline Service worker & 0.489 & 0.312 & 1.630 & 0.884 & 3.007 & 0.118 \\
\hline Agriculture & -0.342 & 0.452 & 0.710 & 0.293 & 1.722 & 0.449 \\
\hline Engineer & -0.270 & 0.585 & 0.763 & 0.242 & 2.405 & 0.645 \\
\hline Simple worker & 0.331 & 0.527 & 1.392 & 0.496 & 3.910 & 0.530 \\
\hline Soldier & -0.656 & 0.739 & 0.519 & 0.122 & 2.208 & 0.375 \\
\hline
\end{tabular}

Variable select method: Stepwise [Forward: Wald].

ings indicate the need for a systematic patient education program including GERD symptoms and aggravating factors. However, according to a previous study, while patient education may increase the degree of disease knowledge, it may not necessarily improve the quality of life of patients with GERD. ${ }^{15}$ Nonetheless, that study had a small sample size and lacked a comparison of therapeutic response. Thus, a larger, multicenter study regarding this issue would be valuable.

Regarding the treatment of GERD, approximately $50 \%$ of patients knew that acid production inhibitors are used to treat GERD, but only $28 \%$ knew that sedatives are not an important treatment for reflux disease, and only $26 \%$ knew that prokinetics are used to treat GERD. These findings show that many Koreans have a relatively low degree of knowledge regarding GERD treatment, which, again suggest the need for a systematic patient education program.

Concerning the prognosis of GERD, $66 \%$ of patients knew that reflux may lead to esophageal ulcers, and $41 \%$ knew that constriction of the esophagus may occur as a consequence of reflux. However, $84 \%$ falsely believed that reflux may cause ulcers in the stomach, and $73 \%$ falsely believed that reflux disease may lead to heart disease. This lack of knowledge may affect quality of life and cause anxiety in patients with GERD. Proper education could be reassuring and improve quality of life.

Statements with lower correct responses $(<30 \%)$ were nervousness is a cause of reflux disease (false), reflux may cause ulcers in the stomach (false), reflux disease may lead to heart disease (false), bloating is a symptom of reflux disease (false), and reflux may lead to increased bile production and esophageal tightening (false). This lack of disease knowledge may delay subjects from seeking treatment until symptoms become severe.

Differences in disease knowledge were evident with respect to age, income, education, and occupation. These findings will be valuable in formulating a systematic GERD educational program. In this study, we found that the educational program desired by many patients is one in which their physician provides a clear and understandable explanation, and which uses a validated and culturally sensitive questionnaire. It seems that the best setting for GERD education is during medical visits and in the community.

Healthcare providers should also educate patients at routine visits regarding the symptoms and treatment of GERD. Education should include identification of risk factors and triggers, symptoms associated with GERD, dietary and life-style modifications for reducing the severity and frequency of symptoms, use of appropriate medications, and guidelines for seeking care. However, the effectiveness of such a GERD educational program has not yet been demonstrated.

In one prior study, a GERD educational program was useful for increasing disease knowledge, but did not improve quality of life in all patients. ${ }^{15}$ For this reason, a well-designed educational program and study in Korea would be valuable. 
This study was limited by the use of a questionnaire that was validated after translation; thus, there might be some bias due to differences in language and culture. In addition, we knew that lack of data regarding the characteristics of the enrolled patients would be a limitation in assessing the factors associated with degree of GERD knowledge. A larger study using a self-developed questionnaire might obtain more precise information.

In conclusion, our study is the largest to date that evaluates the degree of disease knowledge in Korean patients with GERD. The mean degree of GERD knowledge in Korea population is lower than that of Western countries. We believe that this study reflects the real status of GERD knowledge in Korean patients. Because prevalence of GERD in Korea has increased to over 7\%, there is a pressing need for a meaningful and beneficial GERD educational program. The current data should be valuable in formulating such a program.

\section{Supplementary Materials}

Note: To access the supplementary table mentioned in this article, visit the online version of Journal of Neurogastroenterology and Motility at http://www.jnmjournal.org/, and at https://doi. org/10.5056/jnm16123.

Financial support: This work was supported by a grant from The Korean Society of Neurogastroenterology and Motility. Authors had complete access to the data that support the publication.

\section{Conflicts of interest: None.}

Author contributions: In Du Jeong: study concept and design, acquisition of data, analysis and interpretation of data, drafting of the manuscript, and statistical analysis; Moo In Park: study concept and design, acquisition of data, analysis and interpretation of data, study supervision, and critical revision of the manuscript; Sung Eun Kim and Beom Jin Kim: study concept and design, acquisition of data, and analysis and interpretation of data; Sang Wook Kim, Jie-Hyun Kim, Hye Young Sung, and Tae-Hoon Oh: study concept and design, and acquisition of data; and Yeon Soo Kim: study concept and design, acquisition of data, and critical revision of the manuscript. All authors approved the final draft submitted.

\section{References}

1. Yuen E, Romney M, Toner RW, et al. Prevalence, knowledge and care patterns for gastro-oesophageal reflux disease in United States minority populations. Aliment Pharmacol Ther 2010;32:645-654.

2. El-Serag HB, Sweet S, Winchester CC, Dent J. Update on the epidemiology of gastro-oesophageal reflux disease: a systematic review. Gut 2014;63:871-880.

3. Vakil N, van Zanten SV, Kahrilas P, Dent J, Jones R; Global Consensus Group. The Montreal definition and classification of gastroesophageal reflux disease: a global evidence-based consensus. Am J Gastroenterol 2006;101:1900-1920.

4. Dent J, El-Serag HB, Wallander MA, Johansson S. Epidemiology of gastro-oesophageal reflux disease: a systematic review. Gut 2005;54:710717.

5. Wong BC, Kinoshita Y. Systematic review on epidemiology of gastroesophageal reflux disease in Asia. Clin Gastroenterol Hepatol 2006;4: 398-407.

6. Lee SY, Lee KJ, Kim SJ, Cho SW. Prevalence and risk factors for overlaps between gastroesophageal reflux disease, dyspepsia, and irritable bowel syndrome: a population-based study. Digestion 2009;79:196-201.

7. Yang SY, Lee OY, Bak YT, et al. Prevalence of gastroesophageal reflux disease symptoms and uninvestigated dyspepsia in Korea: a populationbased study. Dig Dis Sci 2008;53:188-193.

8. Jung HK. Epidemiology of gastroesophageal reflux disease in Asia: a systematic review. J Neurogastroenterol Motil 2011;17:14-27.

9. Min BH, Huh KC, Jung HK, et al; Functional Dyspepsia Study Group of Korean Society of Neurogastroenterology and Motility. Prevalence of uninvestigated dyspepsia and gastroesophageal reflux disease in Korea: a population-based study using the Rome III criteria. Dig Dis Sci 2014;59:2721-2729.

10. Borgaonkar MR, Townson G, Donnelly M, Irvine EJ. Providing disease-related information worsens health-related quality of life in inflammatory bowel disease. Inflamm Bowel Dis 2002,8:264-269.

11. Ellis SE, Speroff T, Dittus RS, Brown A, Pichert JW, Elasy TA. Diabetes patient education: a meta-analysis and meta-regression. Patient Educ Couns 2004;52:97-105.

12. Griffiths C, Foster G, Ramsay J, Eldridge S, Taylor S. How effective are expert patient (lay led) education programmes for chronic disease? BMJ 2007;334:1254-1256.

13. Kennedy AP, Nelson E, Reeves D, et al. A randomised controlled trial to assess the effectiveness and cost of a patient orientated self management approach to chronic inflammatory bowel disease. Gut 2004;53:1639-1645.

14. Núñez M, Núñez E, Yoldi C, Quintó L, Hernández MV, Muñoz-Gómez J. Health-related quality of life in rheumatoid arthritis: therapeutic education plus pharmacological treatment versus pharmacological treatment only. Rheumatol Int 2006;26:752-757.

15. Urnes J, Farup PG, Lydersen S, Petersen H. Patient education in gastrooesophageal reflux disease: a randomized controlled trial. Eur J Gastroenterol Hepatol 2007;19:1104-1110.

16. Urnes J, Petersen H, Farup PG. Disease knowledge after an educational program in patients with GERD--a randomized controlled trial. BMC Health Serv Res 2008;8:236. 